\title{
Uncertainty and Sensitivity Analyses of Duct Propagation Models
}

\author{
Douglas M. Nark, Willie R. Watson †, and Michael G. Jones ${ }^{\ddagger}$ \\ NASA Langley Research Center, Hampton, VA 23681-2199
}

\begin{abstract}
This paper presents results of uncertainty and sensitivity analyses conducted to assess the relative merits of three duct propagation codes. Results from this study are intended to support identification of a "working envelope" within which to use the various approaches underlying these propagation codes. This investigation considers a segmented liner configuration that models the NASA Langley Grazing Incidence Tube, for which a large set of measured data was available. For the uncertainty analysis, the selected input parameters (source sound pressure level, average Mach number, liner impedance, exit impedance, static pressure and static temperature) are randomly varied over a range of values. Uncertainty limits ( $95 \%$ confidence levels) are computed for the predicted values from each code, and are compared with the corresponding $95 \%$ confidence intervals in the measured data. Generally, the mean values of the predicted attenuation are observed to track the mean values of the measured attenuation quite well and predicted confidence intervals tend to be larger in the presence of mean flow. A two-level, six factor sensitivity study is also conducted in which the six inputs are varied one at a time to assess their effect on the predicted attenuation. As expected, the results demonstrate the liner resistance and reactance to be the most important input parameters. They also indicate the exit impedance is a significant contributor to uncertainty in the predicted attenuation.
\end{abstract}

\section{Nomenclature}

frequency $(\mathrm{Hz})$

$M_{\text {ave }}$

$P_{S}$

$R$

SPL

$T_{S}$

$x_{i}$

Symbols:

$\theta, \chi$

$\theta_{e}, \chi_{e}$

$\mu$

$\sigma$ average mean flow Mach number in the duct

static pressure $(\mathrm{Pa})$

result of a given calculation for specific factor values (e.g., attenuation)

Sound Pressure Level

static temperature $(\mathrm{K})$

$i^{t h}$ factor of a factorial design

normalized liner resistance and reactance, respectively

normalized exit plane resistance and reactance, respectively

mean value

standard deviation

\section{Introduction}

An important aspect of aircraft community and cabin noise is the prediction of engine noise, a primary source of sound from aircraft. The use of acoustic treatment within the engine inlet and aft fan ducts is one of the primary means by which engine noise reduction is achieved. Therefore, one of the main aims of Computational Aeroacoustics (CAA)

* Research Scientist, Structural Acoustics Branch, Research \& Technology Directorate. AIAA Member.

${ }^{\dagger}$ Senior Research Scientist, Computational Aerosciences Branch, Research \& Technology Directorate. AIAA Associate Fellow.

${ }^{\ddagger}$ Senior Research Scientist, Structural Acoustics Branch, Research \& Technology Directorate. AIAA Associate Fellow.

$$
1 \text { of } 13
$$


has been the development of physics-based methodologies to predict the effects of acoustic treatment (as well as other low-noise concepts) on radiated engine noise. Recent years have seen the development of various approaches to modeling sound propagation within complex three-dimensional (3D) aircraft engine nacelle geometries using varying levels of approximation. These include codes based on the convected Helmholtz equation, ${ }^{1-4}$ as well as linearized Euler equation solvers. ${ }^{5-7}$ As described in a companion paper, ${ }^{8}$ an assessment task was undertaken in which a number of these noise prediction and radiation codes were evaluated using a variety of predefined metrics. In particular, a quasi-3D convected Helmholtz solver ${ }^{2}$ (CHQ3D), a linearized Euler solver ${ }^{5}$ (LEE2D-DS), and a convected Helmholtz parabolic approximation ${ }^{1}$ (CDL) code were considered. The assessment was intended to continue a systematic study to identify a "working envelope" within which to use the various approaches. In addition to studying hardwall and fully treated liner configurations, a segmented liner configuration was also considered. The current investigation focuses on an uncertainty analysis performed using the segmented liner configuration. In this case, all selected input parameters were randomly varied across a range of values to investigate the uncertainty in predicted attenuation. Additionally, a subsequent sensitivity analysis that was prompted by the uncertainty results was performed, in which the input parameters were varied one at a time. These analyses provide insight into the influence of the various input parameters on the predicted attenuation values provided by the selected codes.

\section{Code Description}

Although a more detailed description of the codes is provided in the aforementioned companion paper, ${ }^{8}$ a discussion of the underlying formulations is in order. This set of codes represents a wide range of approaches to duct acoustic propagation prediction, including quasi-3D convected Helmholtz and linearized Euler solvers, as well as a parabolic approximation to the convected Helmholtz equation.

\section{A. Quasi-3D Potential Flow Code}

The quasi-3D convected Helmholtz solver ${ }^{2}$ (CHQ3D) utilizes a finite element formulation. The quasi-3D approximation is applicable to duct geometries and flows for which the acoustic solution in one orthogonal direction is separable due to the fact that the sidewalls are both rigid and orthogonal to that direction. The analysis is restricted to a liner impedance that depends only on the axial coordinate and mean velocity fields that have only a uniform axial component. The noise source boundary condition consists simply of constraining all nodal values of acoustic pressure and its derivative at the source plane to the known values. The duct termination condition is also introduced into the system by constraining the nodal degrees of freedom at the duct termination. Effects of the wall impedance boundary condition are included in weak form at the element level by implementation of the Myers ${ }^{9}$ boundary condition.

\section{B. Quasi-3D Rotational Flow Code}

The quasi-3D linearized Euler solver (LEE2D-DS) is based upon a finite element simulation of the linearized equations that govern conservation of mass and momentum for the fluid in the duct. The quasi-3D approximation carries with it the same implications as those discussed previously for the quasi-3D potential flow code, except that more inflow boundary conditions are required. At the inflow (i.e., the source boundary), the acoustic pressure and the two transverse perturbation velocity components are imposed. Across the duct termination plane, a boundary condition is specified relating the acoustic pressure and normal component of acoustic particle velocity through a normalized node impedance matrix. The wall impedance boundary condition remains the Myers boundary condition.

\section{3D Potential Flow Code with Parabolic Approximation}

The three-dimensional potential flow code ${ }^{1}$ (CDL) incorporates the work of Dougherty ${ }^{10,11}$ and utilizes a parabolic approximation to the convected Helmholtz equation. The derivation is based on splitting the solution of the governing equation into 'positive' and 'negative' traveling waves. The terms that couple the equations for the two components are neglected to produce a single parabolic equation for the 'positive' traveling wave. This approach affords very efficient propagation calculations, thus allowing solutions for complex 3D geometries to be handled with relatively low computational costs. The noise source boundary condition consists of constraining the acoustic velocity potential to match the known 'positive' traveling wave source. The solution is then marched from source to exit plane, where no exit impedance is required, as coupling to the 'negative' traveling wave is neglected. The wall impedance boundary condition is obtained by implementation of the Myers boundary condition with modification consistent with the formulation of the parabolic approximation. 
The efficiency obtained by this approximation comes at the expense of reduced accuracy as the direction of propagation of an acoustic mode diverges from the preferred angle of the parabolic approximation. Additionally, loss of accuracy may occur when reflection of acoustic waves in the axial direction becomes important, as these effects are not captured in this formulation. Nevertheless, if appropriate care is taken to account for these limitations, this code can provide an efficient approach to three-dimensional propagation calculations.

\section{Problem Statement}

A rectangular duct geometry was chosen to represent the LaRC Grazing Incidence Tube (GIT), for which high quality experimental data was available to compare with code predictions. This choice allowed connection with a companion uncertainty and sensitivity analysis of impedance prediction models. ${ }^{12}$ In addition, one of the codes is currently only applicable to rectangular geometries.

A detailed description of the GIT apparatus and its capabilities is readily available in the literature. ${ }^{13}$ It has a height and width of $5.08 \mathrm{~cm}$ and, for this study, the length of the computational domain was $60.96 \mathrm{~cm}$. The lower and two side walls are rigid. The upper wall, however, incorporates a hard-soft-hard liner design in which the hard wall segments are $10.16 \mathrm{~cm}$ long and the soft (treated) segment is $40.64 \mathrm{~cm}$ long. The treated segment was a conventional perforate over honeycomb design, the impedance of which was obtained from measurements in the GIT. Sound and flow were directed in the same direction, thus representing aft-engine duct propagation. Further, the centerline Mach numbers ranged from 0.0 to 0.5 , and results were considered for six selected frequencies $(500,1000,1500,2000,2500$ and $3000 \mathrm{~Hz}$ ).

\section{Uncertainty Analysis}

The segmented configuration described above tests the ability of each code (CHQ3D, LEE2D-DS and CDL) to predict the attenuation of a plane wave in a duct containing a uniformly lined segment along the upper wall. For the GIT measurements, six test conditions (two SPL's and three flow rates) were evaluated at frequencies of 500 to $3000 \mathrm{~Hz}$, in steps of $500 \mathrm{~Hz}$. Data were acquired at each of these conditions at least eight times in order to allow for small-sample statistical evaluation. These tests were conducted at different times over a period of one year so that systematic errors due to atmospheric effects could be minimized. Monte-Carlo type simulations were used to obtain statistical metrics for each propagation code. Thirty-one simulations were conducted for each of the six test conditions.

The inputs for each simulation were source SPL, average Mach number, liner resistance and reactance, exit resistance and reactance (when applicable), static pressure and static temperature. Each of these parameters was assumed to vary about their respective mean values, as obtained during GIT tests. ${ }^{12}$ The uncertainty for each input parameter was then assumed to have a Gaussian distribution, the statistics for which are presented in Tables 1, 3 Thus, each of the thirty-one simulations for a particular test condition used inputs randomly selected from Gaussian distributions of the respective input parameters.

Figures 1 and 2 provide comparisons of measured and predicted attenuation bounds. The $95 \%$ confidence intervals for measurements and predictions are provided at each frequency of interest in each figure. The upper and lower values of the $95 \%$ confidence interval for each measurement/prediction method are represented by identical symbols at each frequency. Essentially, this indicates that if another simulation were conducted, there is a $95 \%$ confidence that the result (liner attenuation) would fall between these limits. In general, the mean values of the predicted attenuation are observed to track the mean values of the measured attenuation quite well. It should be noted that the propagation codes assumed a uniform flow profile. As such, it is expected that part of the reason for the differences in the mean predicted and measured attenuation values is due to the absence of the mean flow boundary layer in the predictive methods. In addition, the resonant behavior of this single-degree-of-freedom liner is clearly evident at $1500 \mathrm{~Hz}$ in Figure $1\left(M_{\text {ave }}=0.0, S P L=140 \mathrm{~dB}\right)$. From these results, it can be seen that the differences between predicted and measured SPL attenuations are accentuated at resonance by the choice of single layer liner. When one notes the levels of measured and predicted attenuation at $1500 \mathrm{~Hz}$ of at least $60 \mathrm{~dB}$, these differences between predicted and measured results are of limited concern. Certainly, if a $40.64 \mathrm{~cm}$-long liner provides greater than $60 \mathrm{~dB}$ (predicted or measured), it is performing quite well. It is anticipated that differences between predicted and measured attenuations would be significantly reduced for multi-layer liners, as the resonant behavior of such liners is a less dominant contributor to attenuation. Finally, the data at $1500 \mathrm{~Hz}$ are removed from the accompanying figure in order to allow the results at the remaining frequencies to be more clearly viewed. 
For each flow velocity, the four sets of confidence intervals (one for measured results, three for predictions with different codes) tend to follow the same trends. As expected, the confidence intervals are generally larger with flow than is observed for the no-flow condition.

\section{Sensitivity Analysis}

The Monte-Carlo approach used above assumed that each input parameter was totally uncorrelated with all other input parameters. It is quite possible that increased scatter may result if input conditions deviate from this assumption (i.e., if they are correlated). In light of this, a sensitivity analysis was carried out to investigate the relative importance of each input parameter. In addition to investigating this issue, the sensitivity analysis was also expected to provide further insight into the behavior of the various models near resonance.

A two-level factorial, six factor design as described by Moffat ${ }^{14}$ was used in this portion of the work. In this case, rather than choosing the data points to obtain the most information for the least effort, the statistical design was meant to make best use of the data points to improve the confidence in the inferences drawn from the data. Specifically, the statistical treatment of the data was used to reveal the best estimate of the first derivative and first mixed derivatives of the result with respect to each variable. The analysis was carried out for each of the average Mach numbers $\left(M_{\text {ave }}=0.0,0.25,0.4\right)$ considered in section [IV. Based on observations of this section, the six factors were taken to be the static pressure, static temperature, liner resistance, liner reactance, exit resistance and exit reactance. The upper and lower limits of these factors were considered to be plus or minus two standard deviations about the respective mean values (see Tables 1 3). Only one source SPL (140 dB) was considered, as the codes are linear and are therefore independent of source SPL.

The principle parameters identified in this factorial analysis were the main effect and the interaction effect of each factor with each other factor. The main effect essentially reveals the best estimate of $\left(\frac{\partial R}{\partial x_{i}}\right)$, the volume averaged partial derivative of the result, $R$, with respect to the $i^{\text {th }}$ factor, $x_{i}$. Thus, if the main effect for a selected input parameter (called factor in this analysis) is larger than the main effect for a second input parameter, this indicates the first input parameter is more significant in the computation of the result. The interaction effect, $\left(\frac{\partial^{2} R}{\partial x_{i} \partial x_{j}}\right)$ refers to the variation of the main effect, $\frac{\partial R}{\partial x_{i}}$, for different values of $x_{j}$. There is said to be an interaction if $\frac{\partial R}{\partial x_{i}}$ varies with different values of $x_{j}$. The interaction effect is also referred to as positive if the value of $\frac{\partial R}{\partial x_{i}}$ increases when the value of $x_{i}$ increases. Similar to the discussion for the main effect, if the interaction effect for two selected input parameters is larger than the interaction effect for a second pair of input parameters, this indicates the first pair of input parameters is more significant in the computation of the result. For the current investigation, the result, $R$, of interest was taken to be the attenuation due to the liner, and was calculated as the difference between the sound pressure levels at the computational domain source and exit planes. As mentioned earlier, six input factors $\left(P_{s}, T_{s}, \theta, \chi, \theta_{e}\right.$ and $\left.\chi_{e}\right)$ were evaluated using this approach.

These parameter values for the CHQ3D, LEE2D-DS, and CDL codes are provided in Tables 49 As an example, note the results in Table 4 for the frequencies of 500 and $3000 \mathrm{~Hz}$ at no flow. A comparison of the main effect values at $500 \mathrm{~Hz}$ indicates the liner resistance $(\theta)$ is the most important factor (input parameter) in the computation of attenuation. The reactance at the exit plane $\left(\chi_{e}\right)$ is the next most important factor (about $25 \%$ of the main effect for $\theta$ ), followed by the liner reactance $(\chi)$. The computed attenuation is very weakly dependent on the static temperature $\left(T_{s}\right)$ and the exit plane resistance $\left(\theta_{e}\right)$. By comparison, the results at $3000 \mathrm{~Hz}$ present an entirely different picture. For this condition, four of the factors $\left(\theta, \chi, \theta_{e}\right.$ and $\left.\chi_{e}\right)$ have nearly the same influence on the computation of attenuation, as indicated by the relative values of their main effects. The exit impedance is observed to be slightly more important than the liner impedance for this condition. Table 5 provides the interaction effects for the same conditions (500 and $3000 \mathrm{~Hz}$, no flow). At $500 \mathrm{~Hz}$, the most important combination of input factors is observed to be the liner resistance and reactance $(\theta, \chi)$. However, as its value is quite small (1.4) relative to the main effect values $(69.5,18.3$ and 8.6 for the most important factors), this interaction effect is of limited importance in the computation of attenuation. At $3000 \mathrm{~Hz}$, the interaction effects are observed to grow. For this condition, the most important pair of input factors is the combination of static temperature $\left(T_{s}\right)$ and exit plane reactance $\left(\chi_{e}\right)$. Again, this interaction effect (3.7) is much smaller than most of the main effects (14.8 to 27.4 for the four most important factors); thus, the focus for this condition is on the individual input parameters. 
The attenuation predictions for all codes were not affected by the static pressure. Therefore, main and interaction effects involving this input parameter were all zero. For the CDL code, it can also be seen that the main effect and interaction effect values involving the exit impedance factors (i.e., exit resistance and exit reactance) are all zero. This is to be expected as the current implementation considers only the 'positive' traveling waves and a termination boundary condition is not applied.

Plotting all of the parameters obtained in the analysis would lead to an inordinate number of figures. However, comparisons of the main effect parameter related to the liner impedance for each code at each of the average Mach numbers are provided in Figures 3 through 5 In Figure 3 , the main effect at $M_{\text {ave }}=0.0$ of liner resistance and reactance for the CDL code appear to diverge from those of the CHQ3D and LEE2D-DS codes at and just above resonance (i.e., 1500 and $2000 \mathrm{~Hz}$ ). For the CDL code, the liner resistance has a large effect on the predicted attenuation at 1500 $\mathrm{Hz}$, while the liner reactance has a considerably smaller effect. This trend is reversed at $2000 \mathrm{~Hz}$. The CHQ3D and LEE2D-DS codes show the opposite behavior at these two frequencies. This may be in part due to the difference in liner boundary condition formulation. As the average Mach number increases, the main effect of the liner impedance begins to converge for all of the codes, as seen in Figures 4 and 5 However, a slight variation is evident at $500 \mathrm{~Hz}$ for $M_{\text {ave }}=0.25$.

An alternative approach in which to view these sensitivity results is presented in Figures 6 and 7 Here, the main effect values for the six input factors are presented as a percentage of the total main effect. These percentages are computed by normalizing each main effect value by the summation of the main effect values for all six input factors and subsequently multiplying by 100 . This is done for each code separately at a given frequency and mean flow Mach number, resulting in the relative sensitivity of the predicted attenuation on each input factor. The aforementioned behavior of the codes at resonance under no-flow conditions (i.e., $M_{\text {ave }}=0.0$ ) is illustrated in Figure 6(a) In this case, the main effect due to the liner resistance, $\theta$, makes up approximately $10 \%, 25 \%$, and $90 \%$ of the total sensitivity for the CHQ3D, LEE2D-DS, and CDL codes, respectively. The converse can clearly be seen when considering the liner reactance, $\chi$, which makes up approximately $85 \%, 60 \%$, and $10 \%$ of the overall sensitivity for the CHQ3D, LEE2DDS, and CDL codes, respectively. Figure 6(b) illustrates an interesting aspect of the no-flow predictions at a frequency of $3000 \mathrm{~Hz}$. In this case, the CHQ3D code shows sensitivity to both the exit resistance, $\theta_{e}$, and exit reactance, $\chi_{e}$, while the LEE2D-DS code shows a greater sensitivity to the exit reactance (both suggesting the presence of reflections). As mentioned previously, the CDL code does not incorporate an exit boundary condition and therefore does not exhibit this behavior. Instead, the effect appears to be manifested by an increase in sensitivity due to the liner impedance. Results for $M_{\text {ave }}=0.40$ are presented in Figure 7 and appear to show more consistent sensitivity behavior between the codes at the higher mean flow Mach number (following the trends seen in Figures 4 and 5 ).

Further observation may be drawn from the parameter values in tabular form (Tables 4 9p. The main effect parameter values are provided in Tables 4, 6, and 8 , The static temperature, which is used to determine the sound speed in the duct, tends to decrease in significance as the flow velocity is increased. Although their relative sensitivities vary with frequency and mean flow velocity, the liner impedance components (resistance and reactance) are almost always the most significant (largest main effect values). Overall, the exit impedance components are generally the next most significant, with the dominant effect alternating between the exit resistance and exit reactance.

The interaction effect parameter values are provided in tables 5, 7, and 9 As expected, the interaction between the liner resistance and reactance is observed to be the most significant for almost all test conditions. Interactions between the static temperature and each of the other input parameters are insignificant; especially as the mean flow velocity is increased. Interactions between the exit impedance components and other input parameters tend to be more significant for the LEE2D-DS code than for the CHQ3D code, but are rarely as important as interactions with the liner impedance components. This may be due to the duct termination showing less anechoic behavior as the frequency and/or flow rate increase (i.e., larger influence of reflections from the duct termination).

Overall, the results of this sensitivity analysis have provided valuable insights into the differences between the three propagation codes considered in the current investigation. It seems evident that the use of this type of approach will prove even more valuable as more complex, three-dimensional configurations are considered. Also, it is expected that a statistical design approach (e.g., Modern Design of Experiments) may prove beneficial in limiting the number of cases to be considered in future analysis.

\section{Concluding Remarks}

The codes used in this study were selected because their underlying formulations covered a range of approaches. To quantify the accuracy of these codes, attenuation measurements were made with a conventional perforate-overhoneycomb liner in the NASA Langley Grazing Incidence Tube (GIT). Predicted and measured results were compared 
for a $140 \mathrm{~dB}$ incident sound pressure level, for three flow rates and for source excitation frequencies from 500 to $3000 \mathrm{~Hz}$ in $500 \mathrm{~Hz}$ increments. The predicted attenuations compare well with measured attenuations at low Mach numbers, but tend to diverge with increasing flow Mach number. As expected, the uncertainty (attenuation 95\% confidence interval) is proportional to the amount of attenuation. In other words, small changes in the code inputs at a frequency where large attenuation occurs will result in sizable changes in attenuation.

A sensitivity analysis was also conducted to investigate the relative importance of each input parameter. For this analysis, one parameter was varied at a time. A two-level factorial, six-factor design approach was used to evaluate the influence of individual input parameters (main effect) and pairs of input parameters (interaction effect) on the result of interest (attenuation due to the liner). Generally, each of the codes included in this study showed similar main effect trends for the liner resistance and reactance input factors, except in no-flow conditions at or near the resonant frequency. This difference near resonance for the no-flow condition is likely due to the difference in liner boundary condition formulation between the codes. In terms of interaction effects, the larger influence on the computed attenuation is seen to be the combination of the liner resistance and reactance. In addition, an increase in Mach number generally led to modest increases in the interaction parameter values. This indicates that, as the Mach number increases, the influence of combinations of input parameters on the attenuation also increases.

Overall, this study indicates that an approach to liner design for complex 3D geometries in which codes of varying fidelity are used in a complimentary manner shows great promise. For example, the CDL code could be used to identify preliminary designs and the higher fidelity, more computationally intensive codes (such as 3D versions of the CHQ3D and LEE2D-DS codes) could then be used to refine the results. Future investigations may benefit from considering additional propagation codes to identify possible uncertainty and sensitivity to numerical implementation. Also, as the more complex 3D configurations are considered, use of a statistical design approach (e.g., Modern Design of Experiments) may prove beneficial in limiting the number of cases to consider in future analysis.

\section{References}

${ }^{1}$ Nark, D. M., Farassat, F., Pope, D. S., and Vatsa, V., "The Development of the Ducted Fan Noise Propagation and Radiation Code CDUCTLaRC," AIAA Paper 2003-3242, 2003.

${ }^{2}$ Watson, W. R., Jones, M. G., and Parrott, T. L., “A Quasi-3D Theory for Impedance Eduction in Uniform Flows,” AIAA Paper 2005-2848, 2005.

${ }^{3}$ Eversman, W., "Mapped Infinite Wave Envelope Elements for Acoustic Radiation in a Uniformly Moving Medium," Journal of Sound and Vibration, Vol. 224, 1999, pp. 665-687.

${ }^{4}$ Free Field Technologies, Louvain-la-Neuve, Belgium, MSC.ACTRAN 2005 User's Manual, 2005. 2001.

${ }^{5}$ Watson, W. R., Tracy, M. B., Jones, M. G., and Parrott, T. L., "Impedance Eduction in the Presence of Shear Flow," AIAA Paper 2001-2263,

${ }^{6}$ Watson, W. R., "Three-Dimensional Rectanguar Duct Code with Application to Impedance Eduction," AIAA Journal, Vol. 40, No. 2, 2002, pp. 217-226. 2005.

${ }^{7}$ Lan, J. H. and Breard, C., "Validation of 3D Acoustic Propagation Code with Analytical and Experimental Results," AIAA Paper 2005-2901,

${ }^{8}$ Watson, W. R., Nark, D. M., and Jones, M. G., "Assessment of 3-D Codes for Predicting Liner Attenuation in Flow Ducts," AIAA Paper 2008-2828, 2008.

${ }^{9}$ Myers, M. K., "On the Acoustic Boundary Condition in the Presence of Flow," Journal of Sound and Vibration, Vol. 71, No. 3, 1980, pp. $429-434$.

${ }^{10}$ Dougherty, R. P., “A Wave-Splitting Technique for Nacelle Acoustic Propagation,” AIAA Paper 97-1652, 1997.

${ }^{11}$ Dougherty, R. P., "A Parabolic Approximation for Flow Effects on Sound Propagation in Nonuniform, Softwall, Ducts," AIAA Paper 99 1822, 1999.

${ }^{12}$ Jones, M. G., Parrott, T. L., and Watson, W. R., "Uncertainty and Sensitivity Analyses of a Two- Parameter Impedance Prediction Model," AIAA Paper 2008-2928, 2008

${ }^{13}$ Jones, M. G., Watson, W. R., and Parrott, T. L., "Benchmark Data for Evaluation of Aeroacoustic Propagation Codes with Grazing Flow," AIAA Paper 2005-2853, 2005.

${ }^{14}$ Moffat, R. J., "Planning Experimental Programs - Lecture Notes,” Moffat Thermosciences, Inc., Palo Alto, CA, 1990. 


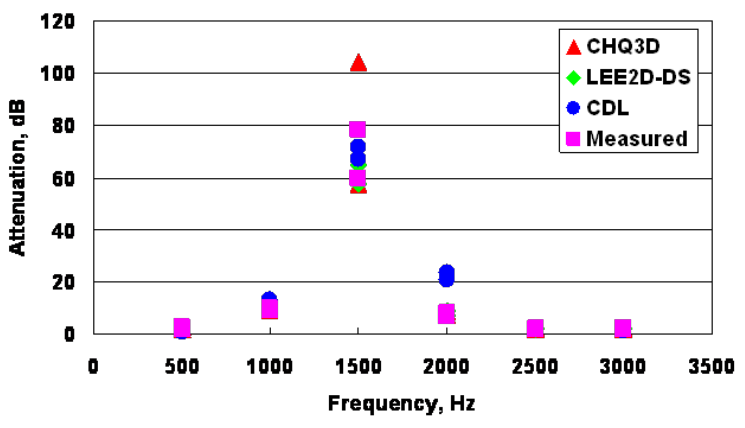

(a) All frequencies

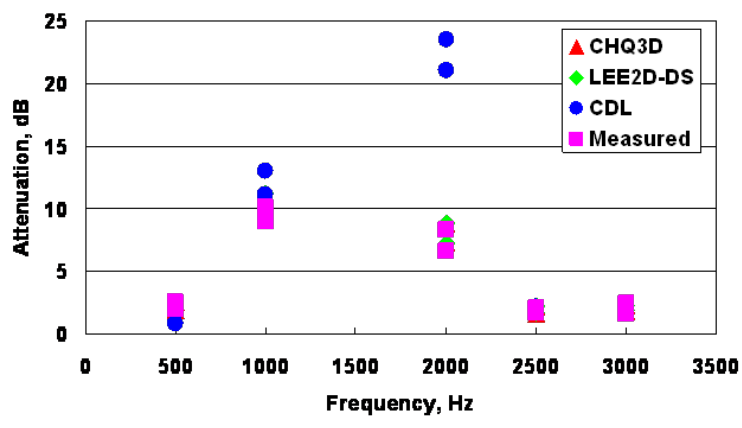

(b) Resonance removed

Figure 1. Results from 9 GIT measurements and 31 simulations for each code; $M_{\text {ave }}=0.0$, Target Source SPL $=140 \mathrm{~dB}$.

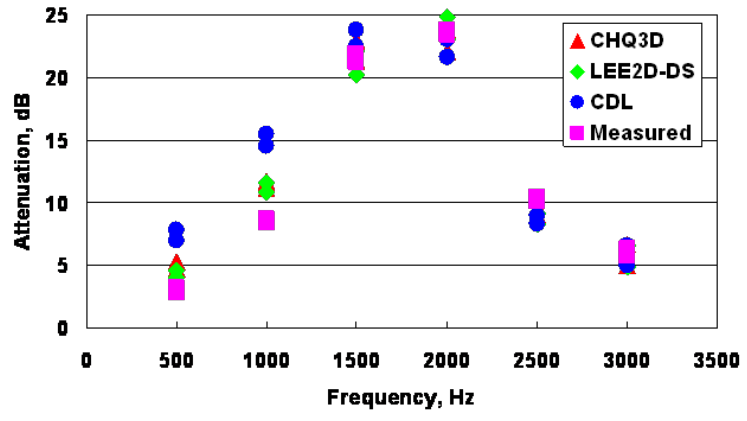

(a) $M_{\text {ave }}=0.25$

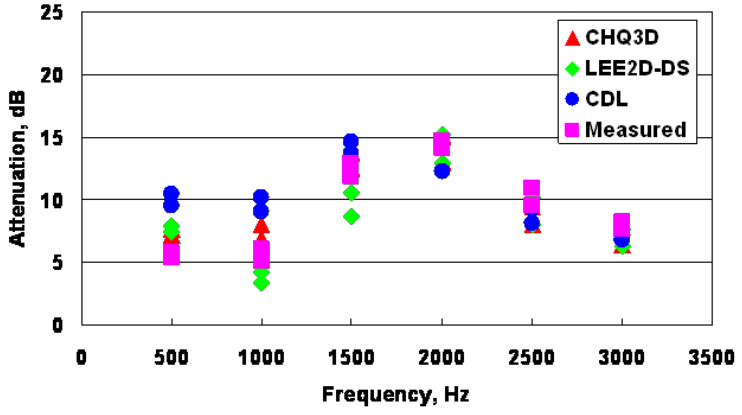

(b) $M_{\text {ave }}=0.4$

Figure 2. Results from 9 GIT measurements and 31 simulations for each code with mean flow, Target Source SPL $=140 \mathrm{~dB}$. 


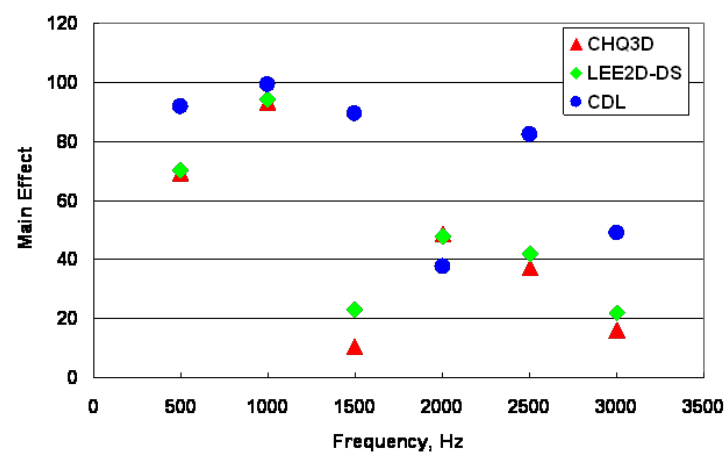

(a) Liner resistance

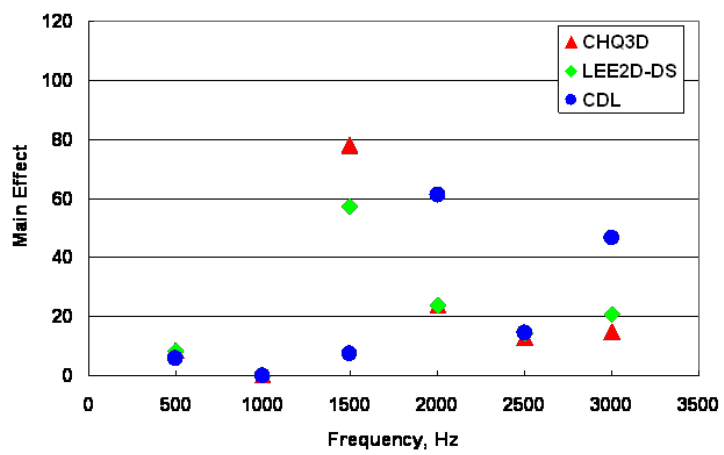

(b) Liner reactance

Figure 3. Main effect parameter values related to liner impedance: $M_{\text {ave }}=0.00$

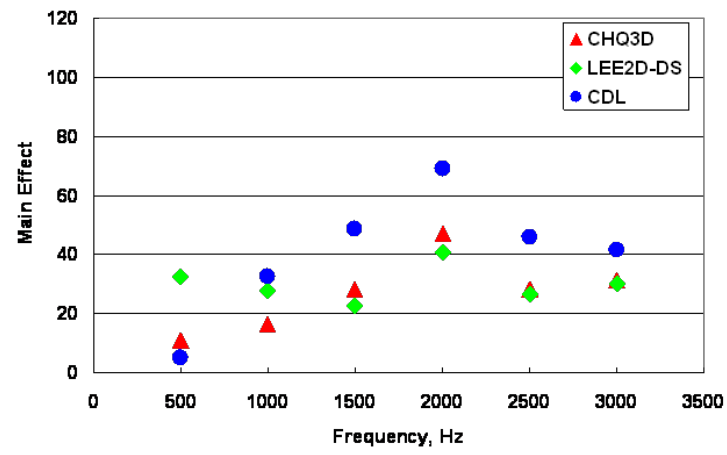

(a) Liner resistance

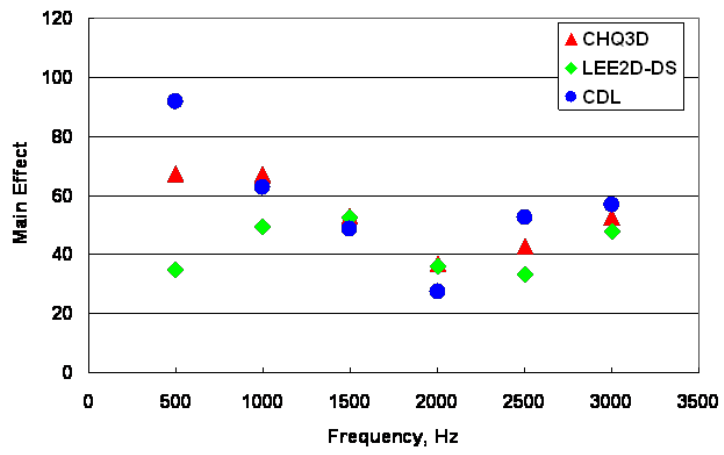

(b) Liner reactance

Figure 4. Main effect parameter values related to liner impedance: $M_{\text {ave }}=0.25$

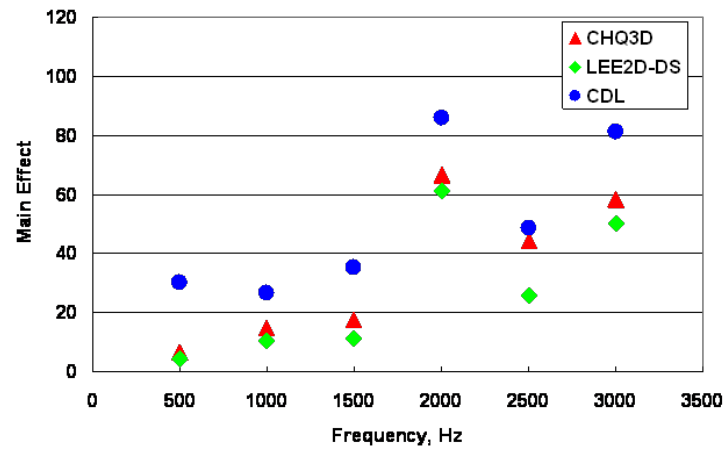

(a) Liner resistance

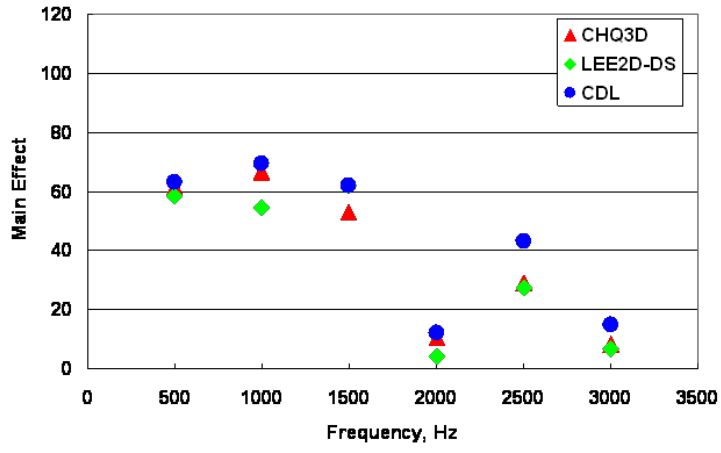

(b) Liner reactance

Figure 5. Main effect parameter values related to liner impedance: $M_{\text {ave }}=0.40$ 


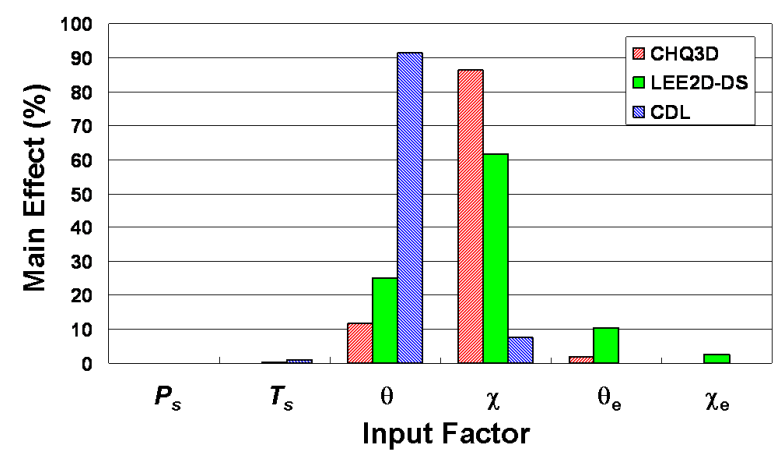

(a) $1500 \mathrm{~Hz}$

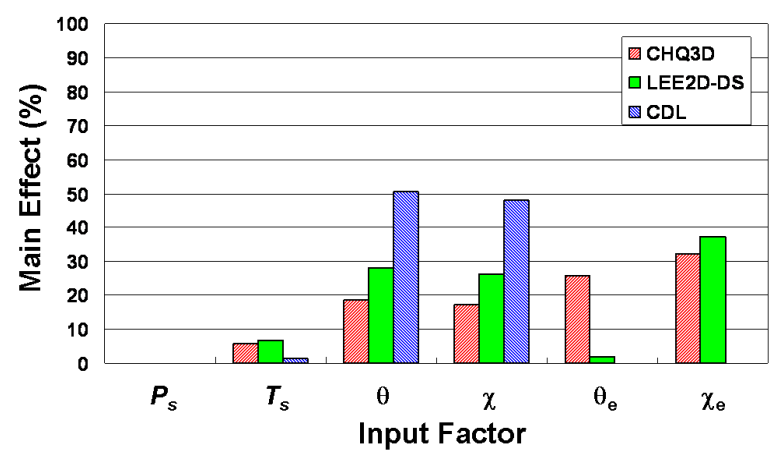

(b) $3000 \mathrm{~Hz}$

Figure 6. Relative sensitivity of the predicted attenuation for each input factor: $M_{a v e}=0.00$. (Note: $P_{s}:$ static pressure, $T_{s}:$ static temperature, $\theta$ : liner resistance, $\chi$ : liner reactance, $\theta_{e}$ : exit resistance, $\chi_{e}$ : exit reactance)

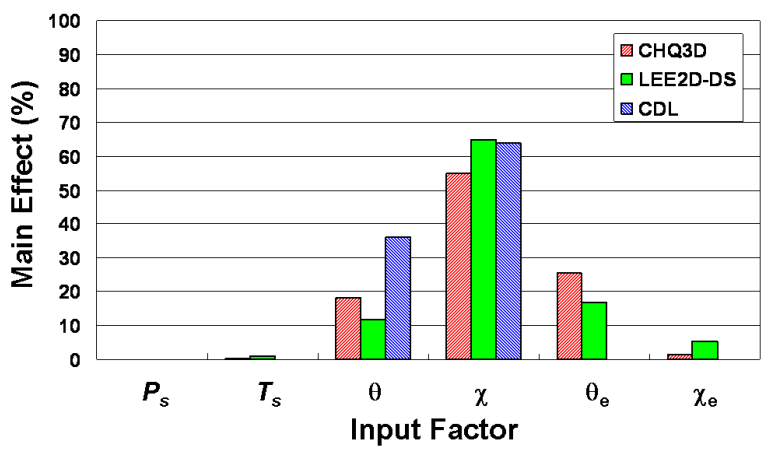

(a) $1500 \mathrm{~Hz}$

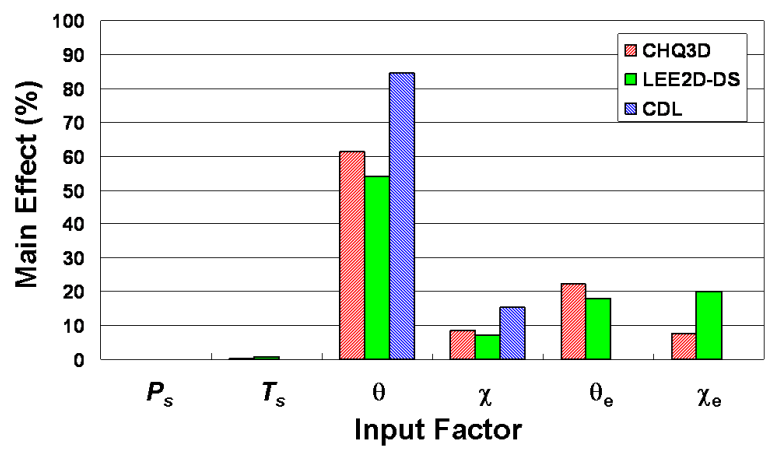

(b) $3000 \mathrm{~Hz}$

Figure 7. Relative sensitivity of the predicted attenuation for each input factor: $M_{\text {ave }}=0.40$. (Note: $P_{s}:$ static pressure, $T_{s}:$ static temperature, $\theta$ : liner resistance, $\chi$ : liner reactance, $\theta_{e}$ : exit resistance, $\chi_{e}$ : exit reactance) 


\begin{tabular}{|c|c|c|c|c|c|c|c|c|}
\hline & \multicolumn{2}{|c|}{$\theta$} & \multicolumn{2}{c|}{$\chi$} & \multicolumn{2}{c|}{$\theta_{\text {exit }}$} & \multicolumn{2}{c|}{$\chi_{\text {exit }}$} \\
\hline$F(\mathrm{~Hz})$ & $\mu$ & $\sigma$ & $\mu$ & $\sigma$ & $\mu$ & $\sigma$ & $\mu$ & $\sigma$ \\
\hline \hline 500 & 0.22 & 0.06 & -2.79 & 0.02 & 0.99 & 0.00 & 0.04 & 0.01 \\
\hline 1000 & 0.23 & 0.02 & -1.03 & 0.00 & 0.99 & 0.01 & 0.05 & 0.00 \\
\hline 1500 & 0.39 & 0.01 & -0.22 & 0.06 & 0.27 & 0.12 & -1.51 & 0.19 \\
\hline 2000 & 0.20 & 0.01 & 0.42 & 0.01 & 0.94 & 0.03 & 0.11 & 0.02 \\
\hline 2500 & 0.21 & 0.01 & 1.00 & 0.01 & 0.96 & 0.03 & 0.07 & 0.02 \\
\hline 3000 & 0.35 & 0.01 & 1.57 & 0.04 & 0.93 & 0.04 & 0.08 & 0.03 \\
\hline \hline & \multicolumn{3}{|c|}{$M_{\text {ave }}$} & \multicolumn{2}{c|}{$P_{S}(\mathrm{~Pa})$} & \multicolumn{2}{c|}{$T_{s}(\mathrm{~K})$} & \multicolumn{2}{c|}{ SPL (dB) } \\
\hline & $\mu$ & $\sigma$ & $\mu$ & $\sigma$ & $\mu$ & $\sigma$ & $\mu$ & $\sigma$ \\
\hline \hline & 0.0 & 0.0 & 101686 & 485 & 295.8 & 0.6 & 140.5 & 1.1 \\
\hline
\end{tabular}

Table 1. Statistical description of input parameters; $M_{\text {ave }}=0.0$, Target $\mathrm{SPL}=140 \mathrm{~dB}$. The values of $M_{\text {ave }}, P_{s}, T_{s}$, and SPL are frequency independent.

\begin{tabular}{|c|c|c|c|c|c|c|c|c|}
\hline & \multicolumn{2}{|c|}{$\theta$} & \multicolumn{2}{c|}{$\chi$} & \multicolumn{2}{c|}{$\theta_{\text {exit }}$} & \multicolumn{2}{|c|}{$\chi_{\text {exit }}$} \\
\hline$F(\mathrm{~Hz})$ & $\mu$ & $\sigma$ & $\mu$ & $\sigma$ & $\mu$ & $\sigma$ & $\mu$ & $\sigma$ \\
\hline \hline 500 & 1.39 & 0.03 & -1.60 & 0.05 & 0.89 & 0.01 & 0.19 & 0.01 \\
\hline 1000 & 1.08 & 0.02 & -0.77 & 0.02 & 0.94 & 0.01 & -0.08 & 0.01 \\
\hline 1500 & 0.86 & 0.01 & -0.35 & 0.02 & 1.11 & 0.04 & -0.03 & 0.03 \\
\hline 2000 & 0.82 & 0.02 & 0.15 & 0.01 & 1.04 & 0.03 & 0.10 & 0.02 \\
\hline 2500 & 0.87 & 0.02 & 0.68 & 0.01 & 0.92 & 0.02 & -0.08 & 0.02 \\
\hline 3000 & 1.20 & 0.13 & 1.15 & 0.09 & 1.04 & 0.03 & -0.11 & 0.03 \\
\hline \hline & \multicolumn{3}{|c|}{$M_{\text {ave }}$} & \multicolumn{2}{c|}{$P_{s}(\mathrm{~Pa})$} & \multicolumn{2}{c|}{$T_{s}(\mathrm{~K})$} & \multicolumn{2}{c|}{ SPL (dB) } \\
\hline & $\mu$ & $\sigma$ & $\mu$ & $\sigma$ & $\mu$ & $\sigma$ & $\mu$ & $\sigma$ \\
\hline \hline & 0.251 & 0.0012 & 101933 & 862 & 295.2 & 0.2 & 140.7 & 0.8 \\
\hline
\end{tabular}

Table 2. Statistical description of input parameters; $M_{\text {ave }}=\mathbf{0 . 2 5}$, Target SPL $=140 \mathrm{~dB}$. The values of $M_{\text {ave }}, P_{s}, T_{s}$, and SPL are frequency independent.

\begin{tabular}{|c|c|c|c|c|c|c|c|c|}
\hline & \multicolumn{2}{|c|}{$\theta$} & \multicolumn{2}{c|}{$\chi$} & \multicolumn{2}{c|}{$\theta_{\text {exit }}$} & \multicolumn{2}{c|}{$\chi_{\text {exit }}$} \\
\hline$F(\mathrm{~Hz})$ & $\mu$ & $\sigma$ & $\mu$ & $\sigma$ & $\mu$ & $\sigma$ & $\mu$ & $\sigma$ \\
\hline \hline 500 & 1.21 & 0.04 & -0.901 & 0.03 & 0.91 & 0.02 & -0.26 & 0.01 \\
\hline 1000 & 1.68 & 0.02 & -0.64 & 0.07 & 0.77 & 0.01 & -0.05 & 0.03 \\
\hline 1500 & 1.15 & 0.01 & -0.43 & 0.03 & 1.03 & 0.06 & 0.22 & 0.01 \\
\hline 2000 & 1.30 & 0.04 & 0.05 & 0.02 & 1.16 & 0.05 & -0.12 & 0.06 \\
\hline 2500 & 1.75 & 0.07 & 0.38 & 0.05 & 0.88 & 0.04 & -0.08 & 0.06 \\
\hline 3000 & 2.32 & 0.11 & 0.15 & 0.03 & 1.00 & 0.08 & 0.11 & 0.03 \\
\hline \hline & \multicolumn{3}{|c|}{$M_{\text {ave }}$} & \multicolumn{2}{c|}{$P_{S}(\mathrm{~Pa})$} & \multicolumn{2}{c|}{$T_{s}(\mathrm{~K})$} & \multicolumn{2}{c|}{ SPL (dB) } \\
\hline & $\mu$ & $\sigma$ & $\mu$ & $\sigma$ & $\mu$ & $\sigma$ & $\mu$ & $\sigma$ \\
\hline \hline & 0.400 & 0.0006 & 101823 & 754 & 293.8 & 0.2 & 140.6 & 1.2 \\
\hline
\end{tabular}

Table 3. Statistical description of input parameters; $M_{\text {ave }}=\mathbf{0 . 4 0}$, Target $\mathrm{SPL}=140 \mathrm{~dB}$. The values of $M_{\text {ave }}, P_{s}, T_{s}$, and SPL are frequency independent. 


\begin{tabular}{|c|c|c|c|c|c|c|}
\hline$M_{\text {ave }}$ & $F(\mathrm{~Hz})$ & $T_{s}$ & $\theta$ & $\chi$ & $\theta_{e}$ & $\chi_{e}$ \\
\hline \hline 0.00 & 500 & 1.0 & 69.5 & 8.6 & 0.0 & 18.3 \\
\hline & 1000 & 0.6 & 93.3 & 0.0 & 5.3 & 0.0 \\
\hline & 1500 & 0.2 & 10.6 & 78.2 & 1.5 & 0.1 \\
\hline & 2000 & 4.3 & 48.8 & 23.9 & 1.5 & 16.1 \\
\hline & 2500 & 11.1 & 37.4 & 12.8 & 20.7 & 10.6 \\
\hline & 3000 & 4.9 & 15.9 & 14.8 & 22.0 & 27.4 \\
\hline \hline 0.25 & 500 & 0.2 & 10.9 & 67.6 & 6.1 & 13.3 \\
\hline & 1000 & 0.2 & 16.6 & 66.9 & 4.4 & 8.1 \\
\hline & 1500 & 0.8 & 28.2 & 52.8 & 8.7 & 5.4 \\
\hline & 2000 & 0.8 & 47.1 & 36.7 & 2.7 & 7.5 \\
\hline & 2500 & 0.9 & 28.3 & 42.8 & 16.6 & 9.8 \\
\hline & 3000 & 0.0 & 31.4 & 52.6 & 6.5 & 5.0 \\
\hline \hline 0.40 & 500 & 0.0 & 6.8 & 61.7 & 11.0 & 15.5 \\
\hline & 1000 & 0.2 & 14.9 & 66.7 & 0.6 & 13.5 \\
\hline & 1500 & 0.3 & 17.7 & 53.0 & 24.5 & 1.4 \\
\hline & 2000 & 0.3 & 66.5 & 10.6 & 11.8 & 8.0 \\
\hline & 2500 & 0.0 & 44.3 & 29.0 & 1.4 & 17.5 \\
\hline & 3000 & 0.3 & 58.5 & 8.3 & 21.1 & 7.2 \\
\hline
\end{tabular}

Table 4. CHQ3D main effect parameter values

\begin{tabular}{|c|c|c|c|c|c|c|c|c|c|c|c|}
\hline$M_{\text {ave }}$ & $F(\mathrm{~Hz})$ & $T_{s}, \theta$ & $T_{s}, \chi$ & $T_{s}, \theta_{e}$ & $T_{s}, \chi_{e}$ & $\theta, \chi$ & $\theta, \theta_{e}$ & $\theta, \chi_{e}$ & $\chi, \theta_{e}$ & $\chi, \chi_{e}$ & $\theta_{e}, \chi_{e}$ \\
\hline \hline 0.00 & 500 & 0.1 & 0.1 & 0.0 & 0.3 & 1.4 & 0.0 & 0.4 & 0.0 & 0.4 & 0.0 \\
\hline & 1000 & 0.5 & 0.0 & 0.1 & 0.0 & 0.0 & 0.2 & 0.0 & 0.0 & 0.0 & 0.0 \\
\hline & 1500 & 0.6 & 0.6 & 0.0 & 0.0 & 7.1 & 0.0 & 0.0 & 0.1 & 0.2 & 0.6 \\
\hline & 2000 & 0.1 & 0.0 & 0.6 & 0.3 & 1.6 & 0.5 & 0.3 & 0.5 & 0.4 & 1.3 \\
\hline & 2500 & 0.4 & 0.2 & 1.5 & 1.7 & 0.6 & 1.4 & 0.2 & 0.3 & 0.9 & 0.2 \\
\hline & 3000 & 0.1 & 0.5 & 2.0 & 3.7 & 0.9 & 0.6 & 0.5 & 2.7 & 1.8 & 2.2 \\
\hline \hline 0.25 & 500 & 0.0 & 0.0 & 0.0 & 0.0 & 1.5 & 0.0 & 0.0 & 0.1 & 0.0 & 0.2 \\
\hline & 1000 & 0.0 & 0.0 & 0.0 & 0.0 & 3.4 & 0.0 & 0.1 & 0.1 & 0.0 & 0.1 \\
\hline & 1500 & 0.0 & 0.0 & 0.0 & 0.0 & 3.3 & 0.1 & 0.0 & 0.1 & 0.1 & 0.6 \\
\hline & 2000 & 0.1 & 0.0 & 0.1 & 0.0 & 4.5 & 0.0 & 0.0 & 0.0 & 0.0 & 0.4 \\
\hline & 2500 & 0.0 & 0.1 & 0.1 & 0.1 & 0.7 & 0.1 & 0.1 & 0.1 & 0.0 & 0.2 \\
\hline & 3000 & 0.0 & 0.0 & 0.1 & 0.1 & 2.3 & 0.6 & 0.2 & 0.2 & 0.4 & 0.5 \\
\hline \hline 0.40 & 500 & 0.0 & 0.0 & 0.1 & 0.0 & 3.6 & 0.2 & 0.2 & 0.3 & 0.1 & 0.6 \\
\hline & 1000 & 0.0 & 0.0 & 0.0 & 0.0 & 3.4 & 0.0 & 0.0 & 0.1 & 0.5 & 0.2 \\
\hline & 1500 & 0.0 & 0.0 & 0.0 & 0.0 & 2.3 & 0.1 & 0.0 & 0.2 & 0.0 & 0.3 \\
\hline & 2000 & 0.0 & 0.0 & 0.0 & 0.1 & 2.0 & 0.0 & 0.1 & 0.0 & 0.0 & 0.5 \\
\hline & 2500 & 0.0 & 0.0 & 0.0 & 0.0 & 6.0 & 0.1 & 0.1 & 0.0 & 0.1 & 1.4 \\
\hline & 3000 & 0.0 & 0.0 & 0.1 & 0.1 & 2.2 & 0.2 & 0.1 & 0.1 & 0.0 & 1.6 \\
\hline
\end{tabular}

Table 5. CHQ3D interaction parameter values 


\begin{tabular}{|c|c|c|c|c|c|c|}
\hline$M_{\text {ave }}$ & $F(\mathrm{~Hz})$ & $T_{s}$ & $\theta$ & $\chi$ & $\theta_{e}$ & $\chi_{e}$ \\
\hline \hline 0.00 & 500 & 0.8 & 70.1 & 8.2 & 0.0 & 18.2 \\
\hline & 1000 & 0.5 & 94.0 & 0.0 & 4.8 & 0.0 \\
\hline & 1500 & 0.3 & 23.2 & 57.4 & 9.8 & 2.4 \\
\hline & 2000 & 4.3 & 48.0 & 24.1 & 2.1 & 16.3 \\
\hline & 2500 & 12.3 & 41.9 & 14.5 & 22.9 & 0.9 \\
\hline & 3000 & 5.3 & 22.1 & 20.8 & 1.4 & 29.6 \\
\hline \hline 0.25 & 500 & 0.3 & 32.6 & 34.9 & 21.0 & 9.4 \\
\hline & 1000 & 1.5 & 27.7 & 49.4 & 3.5 & 13.8 \\
\hline & 1500 & 1.4 & 22.8 & 52.5 & 16.7 & 2.7 \\
\hline & 2000 & 0.9 & 40.8 & 35.9 & 7.9 & 9.2 \\
\hline & 2500 & 0.3 & 26.5 & 33.4 & 18.7 & 19.4 \\
\hline & 3000 & 0.2 & 30.2 & 48.0 & 13.0 & 3.6 \\
\hline \hline 0.40 & 500 & 0.0 & 4.4 & 58.6 & 26.4 & 6.5 \\
\hline & 1000 & 0.6 & 10.7 & 54.4 & 1.3 & 29.3 \\
\hline & 1500 & 1.0 & 11.5 & 62.0 & 16.1 & 5.2 \\
\hline & 2000 & 0.1 & 61.3 & 4.3 & 20.4 & 10.4 \\
\hline & 2500 & 0.2 & 25.8 & 27.5 & 8.5 & 23.5 \\
\hline & 3000 & 0.6 & 50.4 & 6.7 & 16.7 & 18.9 \\
\hline
\end{tabular}

Table 6. LEE2D-DS main effect parameter values

\begin{tabular}{|c|c|c|c|c|c|c|c|c|c|c|c|}
\hline$M_{\text {ave }}$ & $F(\mathrm{~Hz})$ & $T_{s}, \theta$ & $T_{s}, \chi$ & $T_{s}, \theta_{e}$ & $T_{s}, \chi_{e}$ & $\theta, \chi$ & $\theta, \theta_{e}$ & $\theta, \chi_{e}$ & $\chi, \theta_{e}$ & $\chi, \chi_{e}$ & $\theta_{e}, \chi_{e}$ \\
\hline \hline 0.00 & 500 & 0.0 & 0.1 & 0.0 & 0.3 & 1.5 & 0.0 & 0.4 & 0.0 & 0.4 & 0.0 \\
\hline & 1000 & 0.4 & 0.0 & 0.1 & 0.0 & 0.0 & 0.2 & 0.0 & 0.0 & 0.0 & 0.0 \\
\hline & 1500 & 0.1 & 1.1 & 0.1 & 0.0 & 1.6 & 0.0 & 0.1 & 0.1 & 0.1 & 3.6 \\
\hline & 2000 & 0.1 & 0.0 & 0.6 & 0.3 & 1.5 & 0.5 & 0.3 & 0.5 & 0.3 & 1.1 \\
\hline & 2500 & 0.4 & 0.2 & 1.2 & 1.9 & 0.6 & 1.6 & 0.1 & 0.2 & 1.0 & 0.1 \\
\hline & 3000 & 0.1 & 0.6 & 3.1 & 4.4 & 1.3 & 0.8 & 0.7 & 3.8 & 2.4 & 3.8 \\
\hline \hline 0.25 & 500 & 0.0 & 0.1 & 0.0 & 0.0 & 0.3 & 0.3 & 0.2 & 0.4 & 0.5 & 0.0 \\
\hline & 1000 & 0.4 & 0.1 & 0.0 & 0.1 & 2.0 & 0.1 & 0.5 & 0.5 & 0.2 & 0.2 \\
\hline & 1500 & 0.1 & 0.0 & 0.0 & 0.0 & 3.4 & 0.0 & 0.0 & 0.0 & 0.0 & 0.4 \\
\hline & 2000 & 0.2 & 0.0 & 0.1 & 0.0 & 4.5 & 0.1 & 0.1 & 0.0 & 0.0 & 0.3 \\
\hline & 2500 & 0.0 & 0.1 & 0.2 & 0.1 & 0.5 & 0.2 & 0.2 & 0.1 & 0.1 & 0.2 \\
\hline & 3000 & 0.1 & 0.1 & 0.1 & 0.1 & 1.8 & 0.9 & 0.4 & 0.3 & 0.7 & 0.6 \\
\hline \hline 0.40 & 500 & 0.0 & 0.0 & 0.0 & 0.0 & 3.1 & 0.1 & 0.2 & 0.3 & 0.0 & 0.2 \\
\hline & 1000 & 0.0 & 0.1 & 0.0 & 0.1 & 2.1 & 0.1 & 0.1 & 0.1 & 0.6 & 0.6 \\
\hline & 1500 & 0.1 & 0.0 & 0.2 & 0.0 & 2.2 & 0.3 & 0.0 & 0.4 & 0.1 & 0.9 \\
\hline & 2000 & 0.1 & 0.0 & 0.1 & 0.1 & 1.3 & 0.5 & 0.6 & 0.2 & 0.3 & 0.4 \\
\hline & 2500 & 0.6 & 0.1 & 0.1 & 0.2 & 6.1 & 1.1 & 1.4 & 0.7 & 1.1 & 3.1 \\
\hline & 3000 & 0.0 & 0.1 & 0.3 & 0.1 & 0.8 & 0.9 & 0.3 & 0.2 & 0.1 & 3.8 \\
\hline
\end{tabular}

Table 7. LEE2D-DS interaction parameter values 


\begin{tabular}{|c|c|c|c|c|c|c|}
\hline$M_{\text {ave }}$ & $F(\mathrm{~Hz})$ & $T_{s}$ & $\theta$ & $\chi$ & $\theta_{e}$ & $\chi_{e}$ \\
\hline \hline 0.00 & 500 & 0.1 & 91.6 & 5.7 & 0.0 & 0.0 \\
\hline & 1000 & 0.9 & 99.1 & 0.0 & 0.0 & 0.0 \\
\hline & 1500 & 1.2 & 89.5 & 7.4 & 0.0 & 0.0 \\
\hline & 2000 & 0.8 & 37.8 & 61.3 & 0.0 & 0.0 \\
\hline & 2500 & 1.2 & 82.5 & 14.7 & 0.0 & 0.0 \\
\hline & 3000 & 1.4 & 49.1 & 46.6 & 0.0 & 0.0 \\
\hline \hline 0.25 & 500 & 0.0 & 5.0 & 91.8 & 0.0 & 0.0 \\
\hline & 1000 & 0.1 & 32.7 & 62.9 & 0.0 & 0.0 \\
\hline & 1500 & 0.0 & 48.6 & 48.5 & 0.0 & 0.0 \\
\hline & 2000 & 0.3 & 69.1 & 27.6 & 0.0 & 0.0 \\
\hline & 2500 & 1.2 & 46.0 & 52.4 & 0.0 & 0.0 \\
\hline & 3000 & 0.1 & 41.8 & 56.9 & 0.0 & 0.0 \\
\hline \hline 0.40 & 500 & 0.0 & 30.0 & 63.1 & 0.0 & 0.0 \\
\hline & 1000 & 0.0 & 26.5 & 69.3 & 0.0 & 0.0 \\
\hline & 1500 & 0.0 & 35.3 & 61.9 & 0.0 & 0.0 \\
\hline & 2000 & 0.1 & 85.8 & 12.0 & 0.0 & 0.0 \\
\hline & 2500 & 0.2 & 48.7 & 43.1 & 0.0 & 0.0 \\
\hline & 3000 & 0.0 & 81.3 & 14.8 & 0.0 & 0.0 \\
\hline
\end{tabular}

Table 8. CDL main effect parameter values

\begin{tabular}{|c|c|c|c|c|c|c|c|c|c|c|c|}
\hline$M_{\text {ave }}$ & $F(\mathrm{~Hz})$ & $T_{s}, \theta$ & $T_{s}, \chi$ & $T_{s}, \theta_{e}$ & $T_{s}, \chi_{e}$ & $\theta, \chi$ & $\theta, \theta_{e}$ & $\theta, \chi_{e}$ & $\chi, \theta_{e}$ & $\chi, \chi_{e}$ & $\theta_{e}, \chi_{e}$ \\
\hline \hline 0.00 & 500 & 0.0 & 0.0 & 0.0 & 0.0 & 2.6 & 0.0 & 0.0 & 0.0 & 0.0 & 0.0 \\
\hline & 1000 & 0.0 & 0.0 & 0.0 & 0.0 & 0.0 & 0.0 & 0.0 & 0.0 & 0.0 & 0.0 \\
\hline & 1500 & 0.1 & 1.0 & 0.0 & 0.0 & 0.9 & 0.0 & 0.0 & 0.0 & 0.0 & 0.0 \\
\hline & 2000 & 0.1 & 0.1 & 0.0 & 0.0 & 0.0 & 0.0 & 0.0 & 0.0 & 0.0 & 0.0 \\
\hline & 2500 & 0.2 & 0.0 & 0.0 & 0.0 & 1.5 & 0.0 & 0.0 & 0.0 & 0.0 & 0.0 \\
\hline & 3000 & 0.1 & 0.2 & 0.0 & 0.0 & 2.7 & 0.0 & 0.0 & 0.0 & 0.0 & 0.0 \\
\hline \hline 0.25 & 500 & 0.0 & 0.0 & 0.0 & 0.0 & 3.2 & 0.0 & 0.0 & 0.0 & 0.0 & 0.0 \\
\hline & 1000 & 0.0 & 0.0 & 0.0 & 0.0 & 4.3 & 0.0 & 0.0 & 0.0 & 0.0 & 0.0 \\
\hline & 1500 & 0.0 & 0.0 & 0.0 & 0.0 & 2.9 & 0.0 & 0.0 & 0.0 & 0.0 & 0.0 \\
\hline & 2000 & 0.0 & 0.0 & 0.0 & 0.0 & 3.0 & 0.0 & 0.0 & 0.0 & 0.0 & 0.0 \\
\hline & 2500 & 0.0 & 0.0 & 0.0 & 0.0 & 0.4 & 0.0 & 0.0 & 0.0 & 0.0 & 0.0 \\
\hline & 3000 & 0.0 & 0.0 & 0.0 & 0.0 & 1.2 & 0.0 & 0.0 & 0.0 & 0.0 & 0.0 \\
\hline \hline 0.40 & 500 & 0.0 & 0.0 & 0.0 & 0.0 & 6.8 & 0.0 & 0.0 & 0.0 & 0.0 & 0.0 \\
\hline & 1000 & 0.0 & 0.0 & 0.0 & 0.0 & 4.2 & 0.0 & 0.0 & 0.0 & 0.0 & 0.0 \\
\hline & 1500 & 0.0 & 0.0 & 0.0 & 0.0 & 2.8 & 0.0 & 0.0 & 0.0 & 0.0 & 0.0 \\
\hline & 2000 & 0.0 & 0.0 & 0.0 & 0.0 & 2.0 & 0.0 & 0.0 & 0.0 & 0.0 & 0.0 \\
\hline & 2500 & 0.0 & 0.0 & 0.0 & 0.0 & 7.9 & 0.0 & 0.0 & 0.0 & 0.0 & 0.0 \\
\hline & 3000 & 0.0 & 0.0 & 0.0 & 0.0 & 3.8 & 0.0 & 0.0 & 0.0 & 0.0 & 0.0 \\
\hline
\end{tabular}

Table 9. CDL interaction parameter values 